Journal of Mathematics and Informatics

Vol. 7, 2017, 79-86

ISSN: 2349-0632 (P), 2349-0640 (online)

Published 16 May 2017

www.researchmathsci.org

DOI: http://dx.doi.org/10.22457/jmi.v7a10

Journal of

Mathematics and

Informatics

\title{
Testing the Dark Energy Model with Strong Gravitational Lens via a Markov Chain Monte Carlo Approach
}

\author{
Jin-Jun Lu, Yu Pan and Xi-Ming Chen \\ College of Mathematics and Physics \\ Chongqing University of Posts and Telecommunications \\ Nanan-400065, Chongqing, P.R.China \\ E-mail: 1208874237@qq.com
}

Received 20 April 2017; accepted 13 April 2017

\begin{abstract}
We use the markov chain Monte-Carlo method to constrain the dark energy model parameters with 70 strong gravitational lens data(LENs), and combine with baryon acoustic oscillation (BAO), the Planck's cosmic microwave background (CMB) and the type Ia supernovae $(\mathrm{SN})$, where the Strong Gravitational Lens model parameter $f$ is taken as a free parameter. The results of the two combinations of $S N+B A O+C M B$ and $L E N s+B A O+C M B$ show that the limit of dark energy is consistent with the result of $S N$ in the $2 \sigma$ error range. Compared the range of parameters obtained in both $S N+B A O+C M B$ and $S N+L E N s+B A O+C M B$, the strong gravitational lens data can constrain the model parameters more restrictive. In addition, we obtain the strong gravitational lens model parameters $f=1.039_{-0.026}^{+0.030}(1 \sigma)_{-0.035}^{+0.042}(2 \sigma)$, which indicate that the singular isothermal sphere (SIS) model is still an appropriate model for dealing with strong gravitational lens samples.
\end{abstract}

Keywords: strong gravitational lens; dark energy; Markov Chain Monte Carlo algorithm

AMS Mathematics Subject Classification (2010): 28A50

\section{Introduction}

In recent years, astronomical observations of supernovae have shown that the universe is accelerating [1]. So we propose a dark negative pressure to explain the phenomenon of accelerated expansion of the universe. In 2015, the Planck satellite observations gave the current dark energy accounting for $69 \%$ of the universe, but we still do not know what the dark energy is. In order to study its nature, we usually use the combination of theory and observation to discuss the dark energy model. Strong gravitational lens observation becomes one of the important tools for studying cosmology after astronomers observed the first of gravitational lens events in 1979 [2]. Gravitational lens is a phenomenon predicted by Einstein's general theory of relativity. As time and space in the vicinity of large mass objects will be distorted, the light will be curved when it passes through the vicinity of the celestial body. If there is a large mass of foreground objects between the 


\section{Jin-Jun Lu, Yu Pan and Xi-Ming Chen}

observer and the light source, two images will be formed on both sides of the light source. The gravitational lens effect is defined as that there is a lens placed between the observer and the celestial body. The strong gravitational lens effect can magnify the observed celestial bodies (quasars, supernovae, etc.) and has a clean physical background, so it has a great advantage in cosmological research. When the light is propagated in the universe, it will be influenced by the material distribution in the cosmology and the curvature of the universe. Thus we can study the geometric effects of the universe by applying strong gravitational lens data. The data of the strong gravitational lens is cleaner when the problem of the Hubble constant is avoided in terms of cosmic parameter constraints. Thus, compared with other observations, the strong gravitational lens data is more reliable and convincing in terms of the limitations of the cosmic model, and the results are in good agreement with other data $[3,4,5]$.

The Markov chain Monte Carlo algorithm (MCMC) is the most suitable numerical method for the study of cosmology when the dark energy model is observed with astronomical observations. The Markov chain Monte Carlo algorithm is initially applied to computational physics [6], and Hasting's work makes it more general and widely used in space physics, image analysis, and so on. The method was applied to the field of cosmological observation and developed the COSMOMC software package for the study of cosmology. The software has been used to do the relevant work [3,5,8,9]. In this paper, we use the software to achieve 70 sets of gravitational lens samples with the aim at testing the SSLCPL dark energy model.

In this paper, we limit the dark energy by combining 70 strong gravitational lens (LENs) data [10], baryon acoustic oscillations (BAO) [12], Planck cosmic microwave background radiation (CMB) [13], and 580 Ia supernova data (SN) [14]. Generally, we use the singular isothermal sphere (SIS) model to deal with gravitational lens samples and take the parameter $f=1$. However, taking into account the interrelationships with cosmological parameters, we take the gravitational lens model parameter A as a free parameter.

In the second part, we introduce the strong gravitational lens data, the SSLCPL dark energy model and the MCMC algorithm. The third part introduces the limitation method and the result of the strong gravitational lens data on the SSLCPL model. The fourth part is the conclusion.

\section{Data model and method}

\subsection{Strong gravitational lens data}

Geometrically, the gravitational lens phenomenon is determined by the position of the light source, the lens body, and the observer [15].

$o$ is the observer, $s$ is the light source, the angular distance of the lens body from the observer is $D_{d}$, the angular distance of the lens body from the light source is $D_{d s}$, and the angular distance between the observer and the light source is $D_{s} . \hat{\alpha}$ is the deflection angle, $\theta$ is the angle when the ray is linearly propagating, and $\beta$ is the angle of the source to the observer. Since the diameter of the gravitational lens is much smaller than three distances, it can be approximated as a plane. Distance can not be added directly because of the space-time bending effect. 
Testing the Dark Energy Model with Strong Gravitational Lens via a Markov Chain Monte Carlo Approach

When $\beta=0$, that is the light source, the lens and the observer are on the same line, the observed image is ring-shaped, called the Einstein ring. However, based on the limitations of probability and observations, the number of such samples in actual observations is not large. Therefore, we used the maximum angular separation sample of multi-image, and adopted the isothermal sphere (SIS) statistical approximation model.

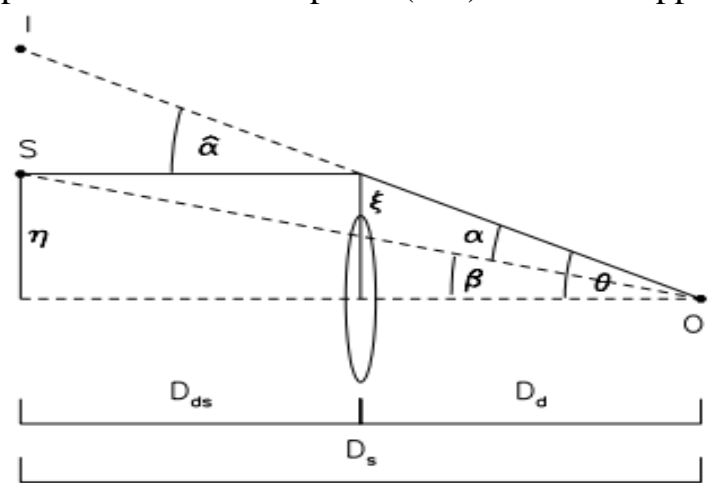

Figure 1: Gravitational lens geometric schematic diagram

The basic assumption of the SIS model is that the motion of stars and other matter components in the galaxy is similar to that of the ideal gas particles, and the spatial distribution of the gravitational potential is spherical symmetry. In the SIS model, the Einstein ring radius $\theta_{E}=4 \pi \frac{\sigma_{S I S}{ }^{2}}{c^{2}} \frac{D_{d s}}{D_{s}}$, Where $c$ is the speed of light, $\sigma_{S I S}$ is the model dispersion velocity. The model parameter $f$ is introduced to describe the relationship with the observed velocity of the stellar velocity $\sigma_{0}$, where $\sigma_{S I S}=f \sigma_{0}$. The range of $f$ is defined as $(0.8)^{1 / 2}<f<(1.2)^{1 / 2}[16]$.

In this paper, we use the 70 sets of distance data obtained in [10] to limit the dark energy model parameters. The theoretical expression of the distance ratio is:

$$
D^{t h}\left(z_{d} ; z_{s} ; p\right)=\frac{D_{d s}}{D_{s}}=\frac{\int_{z_{d}}^{z_{s}} \frac{d z^{\prime}}{E\left(z^{\prime} ; p\right)}}{\int_{0}^{z_{s}} \frac{d z^{\prime}}{E\left(z^{\prime} ; p\right)}},
$$

where $E\left(z^{\prime}, p\right)$ is the parametric dark energy model, $p$ is the cosmological parameters, $z_{s}$ is the source redshift, $z_{d}$ is the redshift of the lens object and the corresponding distance ratio is $D^{o b s}=\frac{c^{2} \theta_{E}}{4 \pi \sigma_{0}^{2} f^{2}}$.

\subsection{Dark energy model}

In the dark energy model, the dark energy state equation $w$ usually has two forms: $w$ is a constant or $w$ evolves with redshift. If we consider that the dark energy equation 


\section{Jin-Jun Lu, Yu Pan and Xi-Ming Chen}

changes with the redshift, we must choose the function of $w(z)$ to determine the parameter change. CPL (Chevallier-Polarski-Linder) parametric model is the most popular dark energy model, where $w(z)=w_{0}+w_{a} \frac{z}{1+z}$. The parameterized Fredman equation is

$$
E^{2}(z ; p)=\Omega_{m}(1+z)^{3}+\left(1-\Omega_{m}\right)(1+z)^{3\left(1+w_{0}+w_{a}\right)} \exp \left(-\frac{3 w_{a} z}{1+z}\right),
$$

where $\Omega_{m}$ is material density parameters and $1-\Omega_{m}$ is dark energy density parameter. Gong studied the association of A and B, and got the relationship between them [11]:

$$
w_{a}=6\left(1+w_{0}\right) \frac{\left(\Omega_{0}^{-1}-1\right)\left[\sqrt{\Omega_{0}}-\tanh ^{-1}\left(\sqrt{\Omega_{0}}\right)\right]}{\Omega_{0}^{-1 / 2}-\left(\Omega_{0}^{-1}-1\right) \tanh ^{-1} \sqrt{\Omega_{0}}},
$$

where $\Omega_{0}$ is the current dark energy density parameter. The simplified dark energy model is called the SSLCPL model.

\subsection{MCMC algorithm}

Before the emergence of the MCMC algorithm, scholars generally used the Bayesian method to analyze the data [17]. With the development of science and technology, scholars need to deal with more data. The relationship between these data is not just a specific function of high-dimensional integral, but more complex high-dimensional integral, and the operation is very difficult to deal with. In this context, the MCMC algorithm based on the Bayesian method was introduced by the scholars in the mid-20th century. On the basis of the Bayesian algorithm, the MCMC algorithm introduces the data into the Monte Carlo algorithm for dynamic analysis through the Markov chain. In this way, it is possible to avoid the shortcomings of the Monte Carlo algorithm that can only be subjected to static analysis, and it can overcome the difficulties of dealing with high-dimensional integrals that can only deal with specific functions.

When taking the MCMC algorithm to process the data, we first construct a Markov chain, and then use the Markov chain to achieve a stable distribution of the effective samples generated Monte Carlo integral [18]. The most important thing for MCMC algorithm is to build the Markov chain, and the most important thing for Markov chain is to construct a suitable transfer nucleus. According to the different transfer kernel, MCMC algorithm will be divided into different types, and the most commonly used are Gibbs sampling [19] and Metropolis-Hastings [20] algorithm.

\section{Analysis}

In this paper, we use the $\chi^{2}$ minimum method to do the observation of SSLCPL dark energy model parameters, and the expression of the $\chi^{2}$ minimum method is

$$
\chi_{\text {LENs }}^{2}(p)=\sum_{i} \frac{\left(D_{i}^{t h}(p)-D_{i}^{o b s}\right)^{2}}{\sigma_{D, i}^{2}} .
$$

Taking into account the correlation between the model parameters and reduce the error, we combined 70 strong gravitational lens, baryon oscillations, cosmic microwave 
Testing the Dark Energy Model with Strong Gravitational Lens via a Markov

Chain Monte Carlo Approach

background radiation and Ia supernova data:

$$
\chi^{2}=\chi_{L E N S}^{2}+\chi_{C M B}^{2}+\chi_{B A O}^{2}+\chi_{S N}^{2} .
$$

We used MCMC algorithm to limit the dark energy model, and got the corresponding parameters of the best fitting value and the corresponding error. The results are showed in Fig. 2.

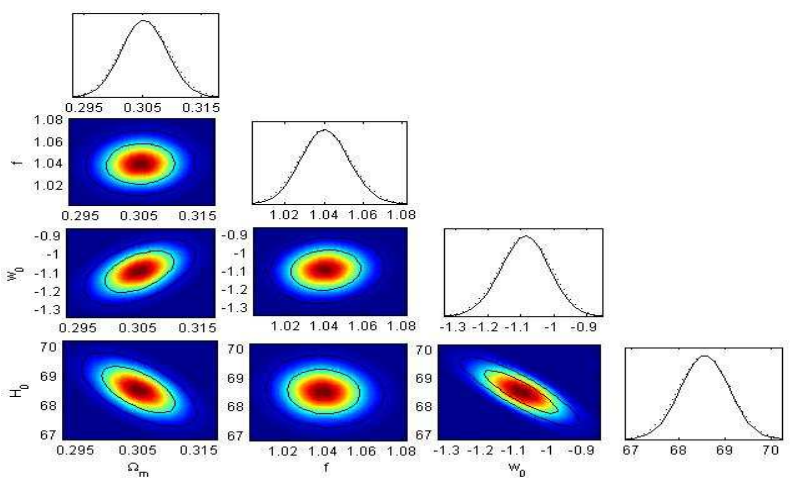

Figure 2: The two-dimensional contours of the parameters under $S N+L E N S+B A O+C M B$ We can see that $f, \Omega_{m}, w_{0}$ and $H_{0}$ are interrelated in Fig. 2. We use $S N+L E N S+B A O+C M B$ to get the $2 \sigma$ error range of the parameters. The results were:

$\Omega_{m}=0.305_{-0.009}^{+0.009}(1 \sigma)_{-0.012}^{+0.012}(2 \sigma)$ (Dark matter density parameter);

$H_{0}=68.560_{-1.186}^{+1.186}(1 \sigma)_{-1.641}^{+1.631}(2 \sigma)$ (Hubble constant);

$w_{0}=-1.088_{-0.071}^{+0.07}(1 \sigma)_{-0.142}^{+0.137}(2 \sigma)$ (Dark energy state equation parameters);

$f=1.039_{-0.026}^{+0.030}(1 \sigma)_{-0.035}^{+0.042}(2 \sigma)$ (SIS model parameters).

In order to study the ability of strong gravitational lens data to limit the SSLCPL dark energy model, the $\mathrm{CMB}+\mathrm{BAO}$ data are taken as priors and compared LENs and SN. Thus, we can get three forms: $S N+L E N s+B A O+C M B$, $S N+B A O+C M B$, and $L E N s+B A O+C M B$. The combination of the different data gives the parameters within the A error range as shown in Table 1.

Table 1: The best fitting parameters and errors of three data combinations

\begin{tabular}{|c|c|c|c|}
\hline $\begin{array}{r}\text { Paramet } \\
\text { er }\end{array}$ & $S N+L E N S+B A O+C M B$ & $S N+B A O+C M B$ & $L E N S+B A O+C M B$ \\
\hline$\Omega_{m}$ & $0.305_{-0.009}^{+0.009}(1 \sigma)_{-0.012}^{+0.012}(2 \sigma)$ & $0.290_{-0.010}^{+0.012}(1 \sigma)_{-0.020}^{+0.023}(2 \sigma)$ & $0.319_{-0.074}^{+0.135}(1 \sigma)_{-0.090}^{+0.186}(2 \sigma)$ \\
\hline$w_{0}$ & $-1.088_{-0.071}^{+0.07}(1 \sigma)_{-0.142}^{+0.137}(2 \sigma)$ & $-1.052_{-0.060}^{+0.052}(1 \sigma)_{-0.110}^{+0.112}(2 \sigma)$ & $-0.737_{-0.31}^{+0.302}(1 \sigma)_{-0.518}^{+0.768}(2 \sigma)$ \\
\hline$H_{0}$ & $68.560_{-1.186}^{+1.186}(1 \sigma)_{-1.641}^{+1.631}(2 \sigma)$ & $69.821_{-2.033}^{+2.121}(1 \sigma)_{-2.738}^{+2.902}(2 \sigma)$ & $66.363_{-10.838}^{+9.771}(1 \sigma)_{-13.653}^{+11.547}(2 \sigma)$ \\
\hline
\end{tabular}

We have the following results by analyzing the data in the table: First, in the $2 \sigma$ error range, the constraints of the three data combinations on SSLCPL satisfy the dark state 


\section{Jin-Jun Lu, Yu Pan and Xi-Ming Chen}

equation parameters. Second, with the LENs data, the parameters are more closely restricted and the error range is reduced. Third, the addition of the LENs data breaks the degeneracy between $w_{0}$ and $\Omega_{m}$. Figures 3-5, the contour lines of the parameters.

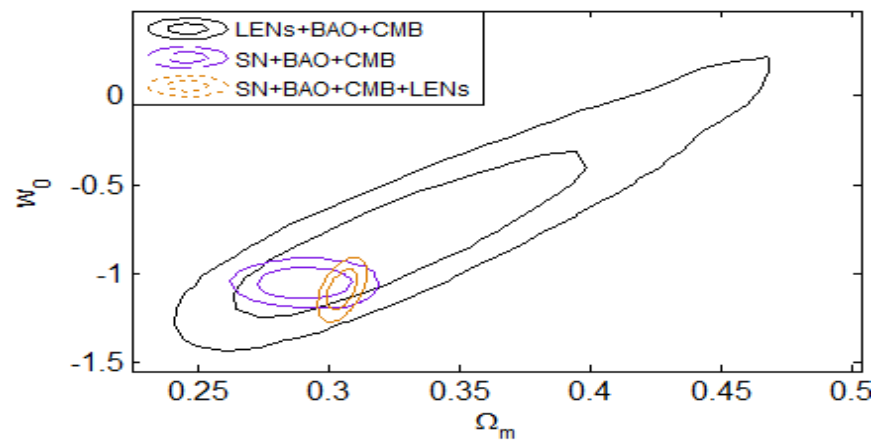

Figure 3: Two-dimensional equal distribution of material density parameter and dark energy state equation

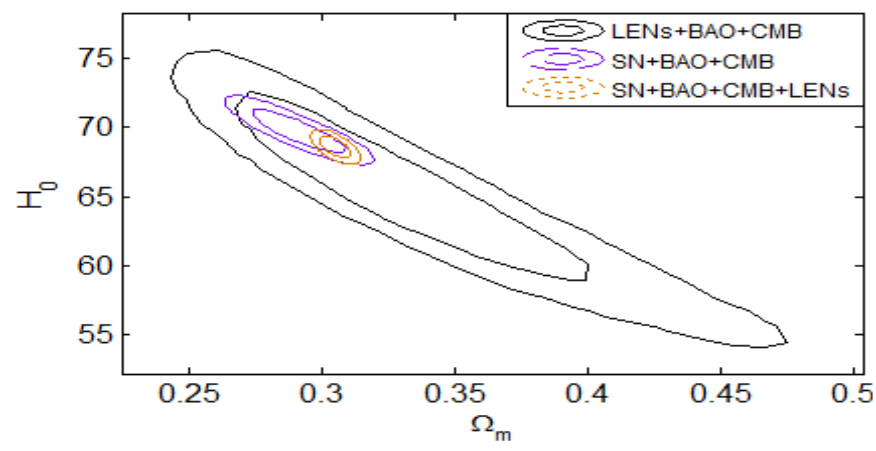

Figure 4: Two-dimensional contour distribution of material density parameters and Hubble constant

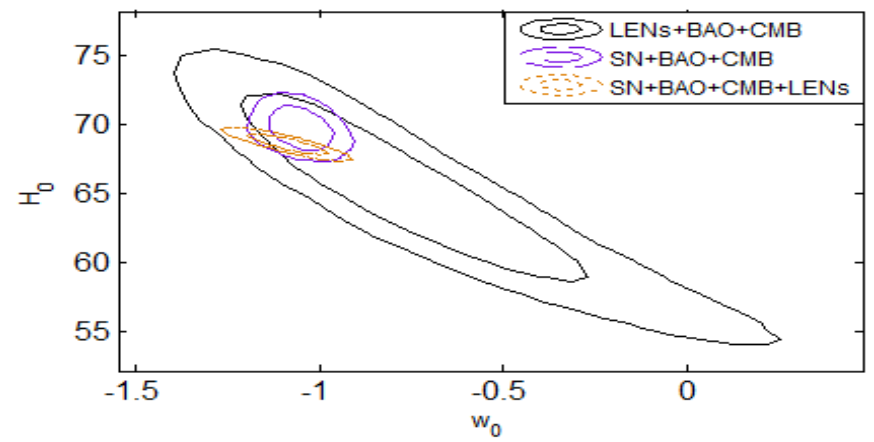

Figure 5: Two-dimensional contour distribution of dark energy state equation parameters and Hubble constant

\section{Conclusion}

In this paper, we mainly take 70 sets LENs and the use of Markov chain Monte Carlo 
Testing the Dark Energy Model with Strong Gravitational Lens via a Markov Chain Monte Carlo Approach

algorithm to limit the SSLCPL dark energy model. We obtain the $2 \sigma$ error range of the parameters. The results are as follows:

$$
\begin{aligned}
& \Omega_{m}=0.305_{-0.009}^{+0.009}(1 \sigma)_{-0.012}^{+0.012}(2 \sigma), H_{0}=68.560_{-1.186}^{+1.186}(1 \sigma)_{-1.641}^{+1.631}(2 \sigma), \\
& w_{0}=-1.088_{-0.071}^{+0.07}(1 \sigma)_{-0.142}^{+0.137}(2 \sigma), f=1.039_{-0.026}^{+0.030}(1 \sigma)_{-0.035}^{+0.042}(2 \sigma) .
\end{aligned}
$$

In order to illustrate the impact of LENs data, we use three different data combinations to limit the SSLCPL dark energy model. We find that: First, in the $2 \sigma$ error range, the constraints of the three data combinations on SSLCPL satisfy the dark state equation parameters. Second, with the LENs data, the parameters are more closely restricted and the error range is reduced. Third, the addition of the LENs data breaks the degeneracy between $w_{0}$ and $\Omega_{m}$. Fourth, The SIS model parameter $f$ in the $2 \sigma$ error range is consistent with the reference [16].

Based on the above results, we can find that it is feasible to use MCMC algorithm and $\chi^{2}$ minimum method to limit the dark energy model of SSLCPL by astronomical observation data. On the other hand, the strong gravitational lens data has some limiting effect on the dark energy model, and the SIS model is still the ideal model for dealing with strong gravitational lens samples.

Acknowledgements. This work is supported by the National Natural Science Foundation of China (No.11447213) and the Scientific and Technological Research Program of Chongqing Municipal Education Commission (Grant No KJ1500414) and Chongqing Municipal Science and Technology Commission Fund (cstc2015jcyj A00044).

\section{REFERENCES}

1. A.G.Riess, A.V.Filippenko, P.Challis, et al, Observational evidence from supernovae for an accelerating universe and a cosmological constant, Astronomical Journal, 116 (3) (1998) 1009-1038.

2. D.Walsh, R.F.Carswell and R.J.Weymann, A, B: twin quasistellar objects or gravitational lens? Nature, 279 (5712) (1979) 381-384.

3. R.Kormann, P.Schneider and M.Bartelmann, Isothermal elliptical gravitational lens models. Astronomy \& Astrophysics, 284 (1994) 285-299.

4. E.Jullo, J.P.Kneib, M.Limousin, et al, A Bayesian approach to strong lensing modelling of galaxy clusters, New Journal of Physics, 9(12) (2007)447-482.

5. S.Cao, C.Giovanni and Z.H.Zhu, Testing the dark energy with gravitational lensing statistics, Astronomical Journal, 755 (2012) 31-45.

6. A.F.M.Smith and G.O.Roberts, Bayesian computation via the gibbs sampler and related markov chain Monte Carlo methods, Journal of the Royal Statistical Society, 55(1) (1993) 3-23.

7. A.Lewis and S.Bridle, Cosmological parameters from CMB and other data: a MonteCarlo approach, Physical Review D, 66(10) (2002) 568-584.

8. X.Wang, M.Tegmark, B.Jain, et al, The last stand before MAP: cosmological parameters from lensing, CMB and galaxy clustering, Physical Review D Particles \& Fields, 68(12) (2003) 634-642. 


\section{Jin-Jun Lu, Yu Pan and Xi-Ming Chen}

9. J.W.Hu, R.G.Cai, Z.K.Guo, et al, Cosmological parameter estimation from CMB and X-ray clusters after Planck, Journal of Cosmology \& Astroparticle Physics, 2014(5) (2014) 628-653.

10. S.Cao, Y.Pan, M.Biesiada, et al, Constraints on cosmological models from strong gravitational lensing systems, Journal of Cosmology \& Astroparticle Physics, 2012(03) (2012) 140-143.

11. Y.G.Gong and Q.Gao, On the effect of the degeneracy among dark energy parameters, The European Physical Journal C, 74(1) (2014) 1-8.

12. Y.Pan, S.Cao and L.Li, Constaints on interacting dark energy from time delay lenses, International Journal of Modern Physics D, 25 (2016) 1-18.

13. Y.Wang and S.Wang, Distance priors from planck and dark energy constraints from current data, Physics Review D. 88 (2013) 476-485.

14. N.Suzuki, D.Rubin, C.Lidman, et al, The hubble space telescope cluster supernova survey v. improving the dark-energy constraints above and building an early-typehosted supernova sample, Astronomical Journal, 746 (2012) 85-102.

15. R.Narayan and M.Bartelmann, Lectures on Gravitational Lensing. Physics, 1997.

16. E.O.Ofek, H.W.Rix and M.Dan, The redshift distribution of gravitational lenses revisited: constraints on galaxy mass evolution, Monthly Notices of the Royal Astronomical Society, 343(2) (2003) 639-652.

17. W.R.Gilks, Markov Chain Monte Carlo, Numerical Analysis for Statisticians, Springer New York, (1999).

18. P.J.Green, Reversible jump Markov chain Monte Carlo computation and Bayesian model determination, Biometrika, 82(4) (1995) 711-732.

19. B.Carlo, G.B.Nicola, R.G.Walter, et al, Dynamic conditional independence models and Markov chain Monte Carlo methods, Journal of the American Statistical Association, 92(440) (1997) 1403-1412.

20. Z.Yang and B.Rannala, Bayesian phylogenetic inference using DNA sequences: a Markov chain Monte Carlo method, Molecular Biology and Evolution, 14(7) (1997) $17-24$. 